\title{
Structural Human Shape Analysis for Modeling and Recognition
}

\author{
Chutisant Kerdvibulvech $^{1}$ and Koichiro Yamauchi ${ }^{2}$ \\ ${ }^{1}$ Rangsit University, 52/347 Muang-Ake, Paholyothin Rd, Lak-Hok, \\ Patum Thani 12000, Thailand \\ chutisant.k@rsu.ac.th \\ ${ }^{2}$ Keio University, 3-14-1 Hiyoshi, Kohoku-ku 223-8522, Japan \\ yamauchi@hvrl.ics.keio.ac.jp
}

\begin{abstract}
Structural human shape analysis is not a trivial task. This paper presents a novel method for a structural human shape analysis for modeling and recognition using 3D gait signatures computed from 3D data. The 3D data are obtained from a triangulation-based projector-camera system. To begin with, 3D structural human shape data which are composed of representative poses that occur during the gait cycle of a walking human are acquired. By using interpolation of joint positions, static and dynamic gait features are obtained for modeling and recognition. Ultimately, structural human shape analysis is achieved. Representative results demonstrate that the proposed 3D gait signatures based biometrics provides valid results on real-world 3D data.
\end{abstract}

Keywords: Structural Human Shape Analysis, Human Walking, 3D Human Body Model, Model Fitting, Modeling, 3D Recognition.

\section{Introduction}

Structural human shape analysis is an interesting topic to explore for many computer scientists around the world. This is because recognition based on human gait and structural human shape analysis has several advantages such as the acquisition of data at a distance from a non-cooperative subject and a characteristic biometrics signature that cannot be faked for a long time [1]. If the habit of walking is changed consciously, the motion seems unnatural and sooner or later a subject returns to his/her natural way of walking. In addition, gait involves not only surface shape, called static features of body parts, but also continuous motion of joints, called dynamic features. Nixon et al. [2] [3] introduced the concept of a total walking cycle according to which the action of walking is similarly assumed as a periodic signal. The gait cycle is assumed as the time interval. Each leg of human has two phases: swing period, when the foot is off the door moving forward to the next step, and stance period, when the foot is in contact with the floor. In the medical field, Murray et al. [4] introduced standard movement patterns of healthy subjects compared to disabled subjects pathologically. For data collection, required markers are attached to anatomical landmarks of human body. They advocate that the pelvic and thorax rotations are essentially variable from one subject to another. Moreover, agent-based simulation of human movement in street networks was discussed in [5] by Jiang and 
Jia. They conclude interestingly that the moving agents of human movement in large street networks are different in their moving behaviour in the fundamental aspects. For this reason, structural human shape analysis is indeed not trivial.

Recently, biometrics modalities with depth information are an interesting resource. As they can apply to many applications, range scanners have obviously become popular increasing the measurement accuracy and speed. It may be true that there are various approaches for 2D and 3D biometrics. Here, Multi-Cam indicates a single or multicamera system and Pro-Cam indicates a projector-camera system. While biometrics approaches using 3D face, finger, ear, and their multimodal data have been presented, gait recognition methods still utilized video sequences. Thus, we attempt to tackle human recognition using $3 \mathrm{D}$ gait biometrics where both the modeling and the test data are obtained in 3D.

Yamauchi et al.'s work discussed about the possibility of recognition of a walking humans in [6]. However, only the initial results were proposed. In this paper, we present a structural human shape analysis method for recognition using 3D gait biometrics from a projector-camera system. A technique for person identification based on 3D body shape and gait is introduced. 3D human body data consisting of representative poses over one gait cycle are captured. 3D human shape model is fitted to the body data using a bottomup approach. Since the body data is dense and it is at a high resolution, we can interpolate the entire gait sequence to fill-in between gait acquisitions. Gait features are usually considered by both dynamic features and static features. By using gait features, the similarity measure is applied for recognition of a subject and his/her pose.

The rest of this paper is clearly structured as follows. Section 2 introduces the framework of structural human shape analysis in detail. After that, in section 3, the experimental results are examined to ensure that the proposed 3D gait signatures based biometrics provides valid results on real-world 3D data. Section 4 ultimately concludes the paper and points to potential future research.

\section{Structural Human Shape Analysis}

To focus on structural human shape analysis, it is important to understand fundamentally the human gait. The truth is that gait consists of two distinct periods. First is a swing phase. This is a phase when the foot does not touch the ground moving the leg forward. Second is a stance phase. This phase is when the foot touches the ground. The gait cycle is expressed by the swing phase and the stance phase. The cycle begins with foot touch which marks the start of the swing phase. The body weight is transferred onto the other leg and the leg swings forward to meet the ground in front of the other foot. The cycle ends with the foot touch. The start of stance phase is when the heel strikes the ground. The ankle flexes to bring the foot flat on the ground and the body weight transferred onto it. The end of stance phase is when the heel leaves the ground.

We use the assumption that there are four measured poses. The model of the human body we used comes from a kinematic tree. The tree consists of 12 segments. The body segment is approximated by a $3 \mathrm{D}$ tapered cylinder which has one free parameter: the cylinder length. It has two degrees of freedom in rotational joints, in the local coordinate system. Upper torso is the root segment, i.e. the parent of lower torso, right upper leg, and left upper leg. Similarly, other segments are linked to parent segments by the rotational joints. The bounding angles of rotational joints are also important. The constraints are enforced in the form of bounding values of the joint angles. Within these 
constraints the model has enough range of movement to represent various poses. The whole body is rotated around three axes and the other segments are rotated around two axes. Here, neck is the fixed segment between head and upper torso, so the neck joint angles are not considered. The articulated structure of the human body has a total of 40 degrees of freedom (DOFs). The pose is described by a 6-D vector, $p$, representing global position and rotation, a 22-D vector, $\mathrm{q}$, representing the joint angles, and a 12-D vector, $r$, representing the lengths of body part. We denote $s$ as the combination of the representative four poses. Joint DOF values concatenated along the kinematic tree define the kinematic pose as a tuple. One of the reasons why we use only 2-DOF rotational joints in this paper is since the 3D tapered cylinder has rotational symmetry along the direction orthogonal to the radial direction.

Figure 1 shows the method overview of structural human shape analysis. To begin with, we measure $3 \mathrm{D}$ human body data by a triangulation-based projector-camera system with human in the walking postures. We build a temporal structural body model, and then extract static dynamic features. Following these data collection we separate the human body data into six regions, and then $3 \mathrm{D}$ human body model is fitted to the segmented body parts in a top-down hierarchy from head to legs. The body model is refined by the Iterative Closest Point algorithm during the optimization process. The output of structural human shape analysis is successfully obtained to compare it against a database.

The intuition behind the principal component analysis (PCA) [7] is to find a set of basis vectors, so that they explain the maximum amount of variance of the data. PCA is applied to determine the coronal axis ( $X$-axis), vertical axis ( $Y$-axis), sagittal axis (Zaxis), and centroid $(O)$ of a human body in the world coordinate system $(O-X-Y$ $-Z$ ), as described in [8]. The constraints are enforced in the form of bounding values of the joint angles. The whole body is rotated around three axes and the other segments are rotated around two axes. Here, neck is the fixed segment between head and upper torso, so the neck joint angles are not considered.

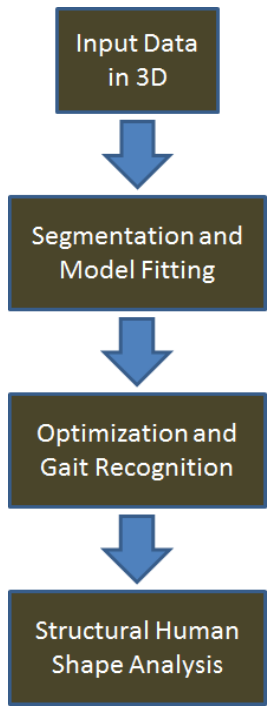

Fig. 1. Method overview of structural human shape analysis 
The combination of the representative four poses is denoted by s. Joint DOF values concatenated along the kinematic tree define the kinematic pose, $\mathrm{k}$, as a tuple, [p, q, r, s], where $\mathrm{p} \in \mathrm{R} 6, \mathrm{q} \in \mathrm{R} 22, \mathrm{r} \in \mathrm{R} 12, \mathrm{~s}=\{\mathrm{s} 1, \mathrm{~s} 2, \mathrm{~s} 3, \mathrm{~s} 4\}$. In the previous works, segments are linked to parent segments by either 1-DOF (hinge), 2-DOF (saddle) or 3-DOF (ball and socket) rotational joints [9]. We use only 2-DOF rotational joints, because the 3D tapered cylinder has rotational symmetry along the direction orthogonal to the radial direction. As a result, we eliminate the twist of body parts as an unnecessary variable.

In our proposed method, we first measure 3D structural human shape data by a triangulation-based projector-camera system with human in the walking postures. Following these data collection we separate the human body data into six regions, and then $3 \mathrm{D}$ human body model is fitted to the segmented body parts in a top-down hierarchy from head to legs. The body model is refined by the Iterative Closest Point algorithm during the optimization process. The human body data are segmented into six regions: head/neck, torso, right arm, left arm, right leg, and left leg. It can be written as $\mathbf{x} 0=\left\{\mathbf{x} 1^{c} \mathbf{X} 2^{c} \mathbf{X} 3^{c} \mathbf{X} 4^{c} \mathbf{X} 5^{c} \mathbf{X} 6\right\}$. The subscript, reg, indicates the region number. Fig. 1 shows body axes, three segments, and six major regions which include a total of twelve body parts. Here, r. and l. indicate right and left, u. and 1. indicate upper and lower (e.g. r. 1. arm is right lower arm). In the following we present a fully automatic parts-based segmentation method.

To begin with, we measure 3D human body data by a triangulation-based projector-camera system with human in the walking postures. Following these data collection we separate the human body data into six regions, and then 3D human body model is fitted to the segmented body parts in a top-down hierarchy from head to legs. The body model is refined by the Iterative Closest Point algorithm during the optimization process. We first consider human body modeling. As discussed in [10], modeling methods usually fail when applied to real data. The real data captured by a projector-camera system have obviously some critical problems. For instance, the projector-camera system cannot cover well particular body parts. The groin region, axillary region, and the side of a human body are some examples. In this way, 3D points of the real data are dependently distributed as explained in [11]. Moreover, the body sways and deep color clothes have detrimental effects which appear as holes and gaps. To solve these mentioned problems, we present a modeling method for dealing with the problems occurring in real data. The proposed method to modeling a walking human incorporates two separate steps. First is model fitting, and second is optimization. The segmentation is useful for coarse registration, because it is unreasonable to fit a kinematic model to articulated objects without any prior knowledge. The prior knowledge allows automatic model fitting and the reduction in the computational cost. Therefore, we fit the model to body data by using the segmented regions. The distance between 3D data of a segmented region, $\mathbf{x} r e{ }^{\wedge} j$, and 3D model of the tapered cylinder, $\mathbf{y}^{i} j$, is linearly minimized. The tapered cylinders can be fitted by determining two angles and one length in the order of levels 1, 2, 3 of the hierarchical structure. With regard to the head and neck, the parameters are estimated from the distribution of $3 \mathrm{D}$ points in the $\mathrm{X}-\mathrm{Y}$ plane and $\mathrm{Y}-\mathrm{Z}$ plane, respectively because the data for hair on the head and lower head region cannot be captured. 
Next step is to get fine registration by minimizing the distance between the body data and kinematic model. The Iterative Closest Point algorithm [12] is utilized in our method. The key steps of the algorithm are: (a) Uniform sampling of points on both shapes. (b) Matching each selected point to the closest sample in the other shape. (c) Uniform weighting of point pairs. (d) Rejecting pairs with distances larger than some multiple of the standard deviation. (e) Point-to-point error metric. (f) Standard selectmatch minimizes iteration.

Obtaining the accurate results depends crucially on the type of gait features. Generally, gait features are divided into two types: (a) dynamic features and (b) static features. For example, the length of stride is one of the significant features of human gait. It can be computed by the leg length and its varying angles between poses. In addition, all the joint positions can be computed by using the same method. Therefore, both dynamic features and static features are used for recognition. We define the dynamic features as joint angles, qm, n, and static features as lengths of the body parts, rm, $\mathrm{n}$. Here, $\mathrm{m}$ is the personal identification number, and $\mathrm{n}$ is the pose index. If dynamic features are only used, a feature vector is defined as $\mathrm{Q}[\mathrm{m}, \mathrm{n}]$. Suppose that unknown feature vector, $\mathrm{Qu}$, is one of $\mathrm{M} * \mathrm{~N}$ feature vectors, $\mathrm{Q}[\mathrm{m}, \mathrm{n}]$. The minimum value of matching scores can be calculated as explained. The matching score is computed as distance. For unknown data, the personal identification number and pose index are recognized.

\section{Experimental Results}

The experiments were performed on the body data set collected by the human body measurement system. It contains twenty-four body data from the representative four poses of six subjects $X \in\{A, B, C, D, E, F\}$. The body data of representative poses are captured by a human body measurement system. The system consists of nine projector-camera pairs, which acquires nine range data in 2 seconds with $640 \times 480$ pixels, $3 \mathrm{~mm}$ depth resolution, and $3 \mathrm{~mm}$ measurement accuracy.

3D structural human shape data of walking humans of Subject A, Subject B, Subject C, Subject D, Subject E, and Subject F are depicted respectively. The number of measuring points is about $1 / 2$ to one million depending on the subject and the pose. The full results of gait reconstruction are also experimented. It is defined that the one gait cycle is composed of twenty frames. The speed is given by dividing the stride length by the number of poses and the direction is not given automatically. The frame index 1, 6, 11, and 16 are representative poses, while the other frame indexes are calculated poses. The representative poses and their symmetric poses are used for the experiment. The poses are symmetric. They are synthesized by allocating right (or left) side parameters of representative poses to left (or right) side parameters of symmetrical poses. Figure 2 shows the results of human body modeling. Similarly, the six subjects of Subject A, Subject B, Subject C, Subject D, Subject E, and Subject $\mathrm{F}$ are used. The 3D human body model is fitted to four different walking poses. Thus, it contains a total of 24 different poses. The body model is suitably fitted to the captured body data. The joint angles are then obtained symmetrical poses, including the lengths of body parts. 


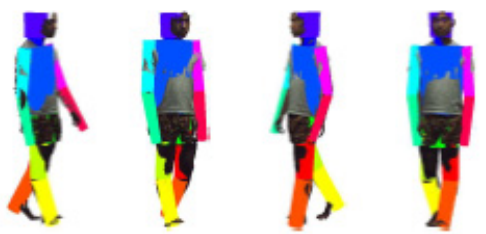

Subject A
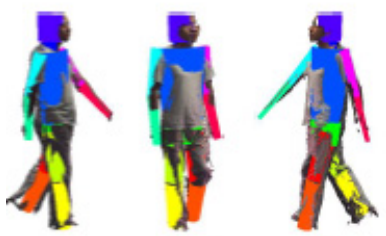

Subject C
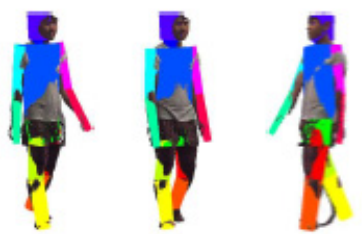

Subject E

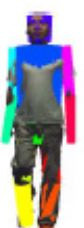

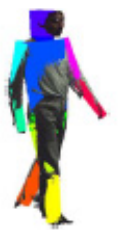
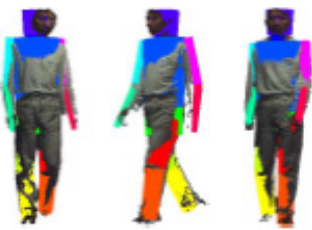

Subject B
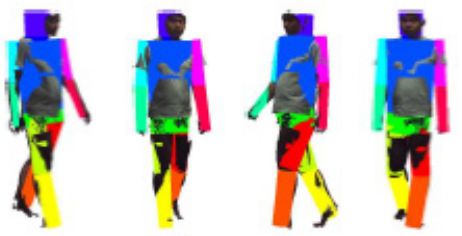

Subject D
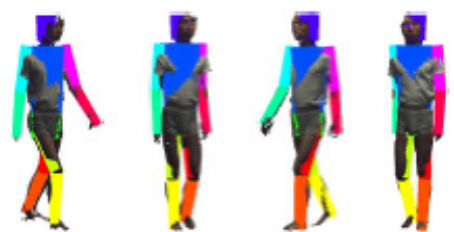

Subject F

Fig. 2. 3D structural human shape data of walking humans fitted to four poses

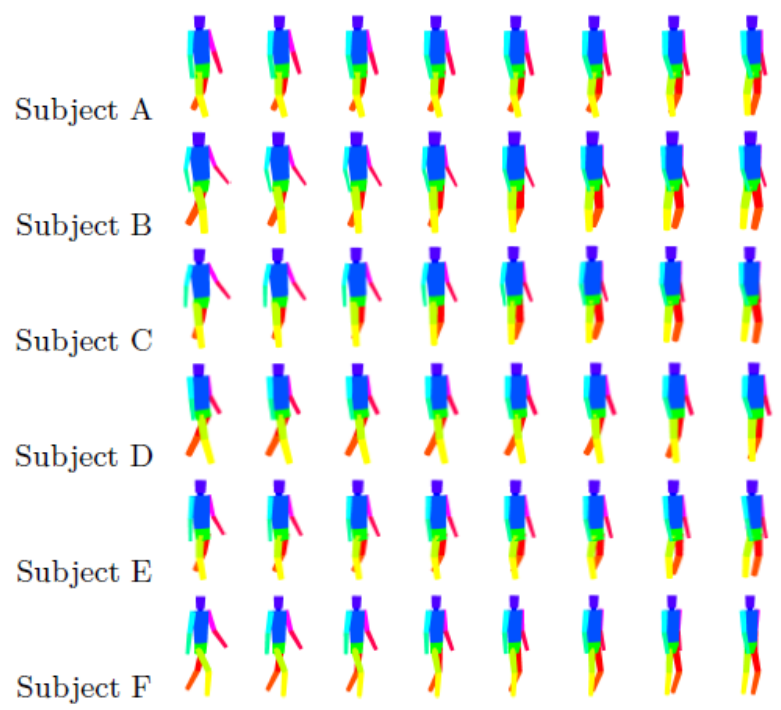

Fig. 3. Representative training data for six subjects 
Figure 3 represents the training data of six subjects, i.e. Subject A, Subject B, Subject C, Subject D, Subject E, and Subject F. Each subject has 40 poses, so that training data contains a total of 240 kinematic poses. For the testing data, one gait sequence is recovered by representative poses $=\{\mathrm{s} 1, \mathrm{~s} 2, \mathrm{~s} 3, \mathrm{~s} 4\}$. Average pose error is calculated in order to estimate the quantitative accuracy of the proposed method. The identification rate is obtained by dividing the number of recognized subjects by the number of testing data. The pose error is the frame difference between the estimated pose and the ideal pose. From our experiment, although bodies for these two subjects are different, their joint angles, i.e. leg and arm swings, are quite similar. For the average pose error we achieve 0.41 frame using dynamic features and 1.31 frames using both features. The experiment using dynamic features has better results, because it focuses on estimating poses, i.e. dynamic features do not consider lengths of body parts. However, by using both features, it definitely provides better results.

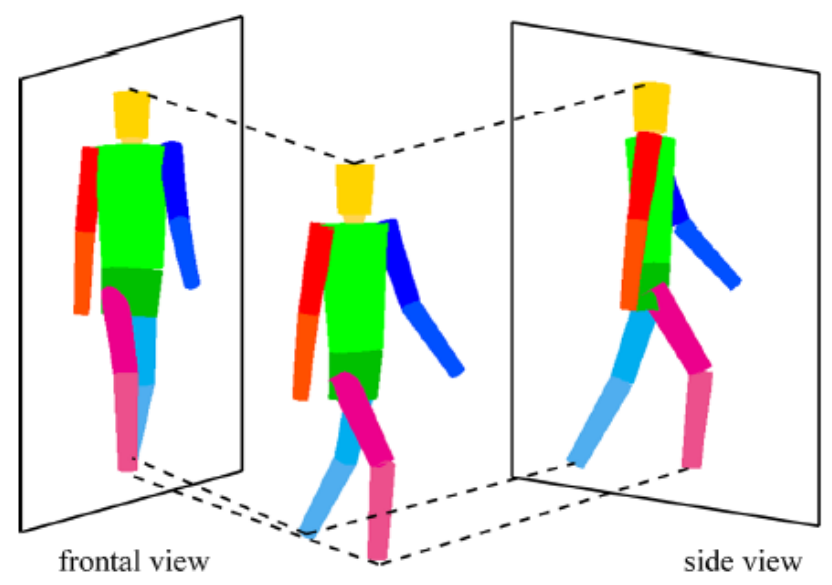

Fig. 4. Virtual front and side views

In fact, gait recognition methods usually use only a single view. This means that the information of 2D gait biometrics is solely used. Figure 4 shows how to compare $3 \mathrm{D}$ gait biometrics with $2 \mathrm{D}$ gait biometrics. Frontal view and side view are synthesized from our data by orthographic projection. We then use 2D gait biometrics for the experiment. From our experimental results, we achieve 95.83 percent using dynamic feature and 100 percent using both dynamic and static features for the identification rate. Furthermore, we achieve 0.57 using dynamic feature and 1.29 using both features for the average pose error. When only dynamic feature is used, the method fails to recognize testing data Subject B with pose 4, Subject D with pose 7, Subject D with pose 8 , and Subject D with pose 14 who should not be recognized as the training data for Subject $C$ with pose 4 , Subject A with pose 7, Subject A with pose 8, and Subject B with pose 13. 
Table 1. Average pose error for frontal view and side view

\begin{tabular}{|l|c|c|}
\hline & Frontal View (\%) & Side View (\%) \\
\hline $\begin{array}{l}\text { Lower Average Pose Error for 3D } \\
\text { Gait Biometrics }\end{array}$ & 29 percent & 72 percent \\
\hline
\end{tabular}

Furthermore, our experiments show that 3D gait biometrics provide lower average pose error. As shown in Table 1, it is 29 percent of the frontal view lower than that result of $2 \mathrm{D}$ gait biometrics. The number is also 72 percent of the side view lower than that of 2D gait biometrics. We believe that these numbers are suitable enough to make this proposed method newly useful for structural human shape analysis for modeling and recognition.

\section{Conclusions}

In this paper, an approach for a structural human shape modeling based on 3D gait biometrics and recognition where the input data are obtained from a triangulationbased projector-camera system is presented. By separating the human body into six regions, a bottom-up approach is utilized to fit the 3D human body. After that, the entire gait sequence is recovered. Finally, both static and dynamic gait features are obtained for recognition task. Our database we used consists of a total of twenty-four body data. In each data, it is comprised of four poses of six subjects. The size of database, we believe, is still quite small. We intend to expand our database to collect a huge number of subjects for possible better results. After that, we intend to apply the proposed method to use in related-applications. In fact, it is applicable to various kinds of computer science fields. We plan to refine this problem in the future.

\section{References}

1. Bhanu, B., Han, J.: Human Recognition at a Distance in Video. Springer, London (2011)

2. Nixon, M.S., Tan, T., Chellappa, R.: Human identification based on gait. Springer, New York (2005)

3. Nixon, M.S., Carter, J.N.: Automatic recognition by gait. Proc. of the IEEE 94(11), 2013-2024 (2006)

4. Murray, M.P., Drought, A.B., Kory, R.C.: Walking patterns of normal men. Journal of Bone and Joint Surgery 46A(2), 335-360 (1964)

5. Jiang, B., Jia, T.: Agent-based Simulation of Human Movement Shaped by the Underlying Street Structure. International Journal of Geographical Information Science 25(1) (2011)

6. Yamauchi, K., Bhanu, B., Saito, H.: Recognition of walking humans in 3D: Initial results. In: Proc. of the IEEE Conference on Computer Vision and Pattern Recognition Workshops (CVPR Workshops), June 20-25, pp. 45-52 (2009)

7. Jolliffe, I.T.: Principal Component Analysis. Springer, New York (2002) 
8. Kerdvibulvech, C., Saito, H.: Real-Time Guitar Chord Recognition System Using Stereo Cameras for Supporting Guitarists. Transactions on Electrical Engineering, Electronics, and Communications (ECTI) 5(2), 147-157 (2007)

9. Vondrak, M., Signal, L., Jenkins, O.C.: Physical simulation for probabilistic motion tracking. In: Proc. of the IEEE Conference on Computer Vision and Pattern Recognition (CVPR), pp. 1-8 (2008)

10. Yu, H., Qin, S., Wight, D.K., Kang, J.: Generation of 3D human models with different levels of detail through point-based simplification. In: Proc. of the International Conference on "Computer as a Tool", pp. 1982-1986 (2007)

11. Werghi, N., Rahayem, M., Kjellander, J.: An ordered topological representation of 3D triangular mesh facial surface: concept and applications. EURASIP Journal on Advances in Signal Processing 1, 1-20 (2012)

12. Rusinkiewicz, S., Levoy, M.: Efficient variants of the ICP algorithm. In: Proc. of the 3-D Digital Imaging and Modeling, pp. 145-152 (2001) 